Revue internationale P.M.E.

Économie et gestion de la petite et moyenne entreprise

\title{
Rôle des partenaires des PME/PMI dans l'amélioration de leurs performances environnementales
}

\section{Natacha Gondran et Christian Brodhag}

Volume 16, numéro 2, 2003

URI : https://id.erudit.org/iderudit/1008438ar

DOI : https://doi.org/10.7202/1008438ar

Aller au sommaire du numéro

Éditeur(s)

Presses de l’Université du Québec

ISSN

0776-5436 (imprimé)

1918-9699 (numérique)

Découvrir la revue

Citer cet article

Gondran, N. \& Brodhag, C. (2003). Rôle des partenaires des PME/PMI dans

l'amélioration de leurs performances environnementales. Revue internationale P.M.E., 16(2), 35-59. https://doi.org/10.7202/1008438ar
Résumé de l'article

Les outils traditionnels de régulation des externalités environnementales (approches réglementaire, économique et contractuelle) reposent sur le postulat que les entreprises sont en situation d'information parfaite en ce qui concerne les contraintes qu'elles doivent respecter, leurs coûts

environnementaux et les mesures qu'elles peuvent prendre pour satisfaire aux exigences qui leur sont imposées. Cependant, une enquête, réalisée auprès de 47 PME, montre que ce postulat n'est pas toujours vérifié, ce qui limite l'efficacité de ces instruments de régulation. Elle met également en évidence le fait que l'information n'est pas une condition suffisante à l'amélioration des performances environnementales des entreprises : certaines d'entre elles profitent de l'asymétrie d'information portant sur leurs performances environnementales pour adopter un comportement de type opportuniste. En revanche, elle révèle que plus une entreprise est ouverte sur ses partenaires, mieux elle intègre l'environnement. 


\title{
Rôle des partenaires des PME/PMI dans l'amélioration de leurs performances environnementales
}

\author{
Natacha GONDRAN \\ Christian BRODHAG \\ École nationale supérieure des mines de Saint-Étienne
}

MOTS CLÉS

Performance environnementale - PME - Accès à l'information

\begin{abstract}
RÉSUMÉ
Les outils traditionnels de régulation des externalités environnementales (approches réglementaire, économique et contractuelle) reposent sur le postulat que les entreprises sont en situation d'information parfaite en ce qui concerne les contraintes qu'elles doivent respecter, leurs coûts environnementaux et les mesures qu'elles peuvent prendre pour satisfaire aux exigences qui leur sont imposées. Cependant, une enquête, réalisée auprès de 47 PME, montre que ce postulat n'est pas toujours
\end{abstract}

\section{LES AUTEURS}

NATACHA GONDRAN, ingénieure de l'Institut national des sciences appliquées de Lyon et docteure en science et technique du déchet, est ingénieure de recherche à l'Ecole des mines de SaintÉtienne. Sa thèse a été financée par l'ADEME (Agence de l'environnement et de la maîtrise de l'énergie). École nationale supérieure des mines de Saint-Étienne, Centre SITE, 158, Cours Fauriel, 42023 Saint-Étienne, cedex 2, France. Courriel : <natacha.gondran@emse.fr>.

Christian BRodHAG, ingénieur civil des Mines et docteur en science. II est directeur de recherche à l'École des mines de Saint-Étienne, ancien président de la Commission française du développement durable, membre du Conseil scientifique de la DATAR (Délégation à l'aménagement du territoire et à l'action régionale) et président du groupe de travail de l'AFNOR sur le développement durable. École nationale supérieure des mines de Saint-Étienne, Centre SITE, 158, Cours Fauriel, 42023 Saint-Étienne, cedex 2, France. Courriel : <brodhag@emse.fr>.

Nous remercions l'ADEME, qui a financé la thèse de Natacha Gondran, ainsi que les partenaires qui nous ont permis de réaliser cette étude : le Parc naturel régional du Pilat, la cellule environnement de la CCI de Pau, Michel Lagoutte et les élèves du BTS environnement de l'ISEME (Institut supérieur européen des métiers de l'environnement) de Fontenay-leComte, ainsi que Frédéric Poulard, élève-ingénieur de l'ENS des mines de Saint-Étienne et la CCl de Saint-Étienne Montbrison.

Nous remercions également deux rapporteurs anonymes de la Revue internationale PME pour leurs remarques constructives sur ce texte. 
vérifié, ce qui limite l'efficacité de ces instruments de régulation. Elle met également en évidence le fait que l'information n'est pas une condition suffisante à l'amélioration des performances environnementales des entreprises: certaines d'entre elles profitent de l'asymétrie d'information portant sur leurs performances environnementales pour adopter un comportement de type opportuniste. En revanche, elle révèle que plus une entreprise est ouverte sur ses partenaires, mieux elle intègre l'environnement.

\begin{abstract}
Traditional regulatory and economic mechanisms that are aimed to regulate environmental externalities assume that firms are totally informed about their regulatory constraints, their environmental costs and about the solutions that they can implement to satisfy these exigencies. A survey on 47 SMEs shows that this assumption is not always verified. Thus, the efficacy of these regulation mechanisms is limited. Information is not sufficient: some SMEs may be opportunistic because of information asymmetry between stakeholders and the firm about its environmental impacts. However, the survey shows a link between SME's openness to its stakeholders and its environmental performances. It appears that the main sources of information of the firm are the partners with whom the firm has a direct contact.
\end{abstract}

\title{
RESUMEN
}

Los instrumentos tradicionales de regulación de las externalidades ambiental estipulan a que las empresas reguladas estan en una situación de perfecta información de sus obligaciones, de los costes ambientales y de las soluciones que ellas pueden implementar para complir con las leyes. Sin embargo, una encuesta realisada en 47 PyMEs muestra que esta regla no es siempre complida. Lo que limita la eficiencia de los instrumentos tradicionales de regulación ambiental (por ejemplo: leyes, fiscalidad, etc.). Se observa también que el nivel de integración del medio ambiente en la gestión de la empresa esta relacionado a la apertura de la empresa sobre otros organismos profesionales. La encuesta también evidencía un comportamiento oportunista de parte de algunas empresas que aprovechan de la situación de asimetria de información con respecto a sus impactos ambiental.

\section{ZUSAMMENFASSUNG}

Die traditionellen Werkzeuge der Regulierung der Umweltexternalitäten (vorgeschriebene, wirtschaftliche und vertragliche Konzepte), beruhen auf dem Postulat, dass die Unternehmen vollkommenen informiert sind über die einzuhaltenden Umweltauflagen, über die geforderten Lösungen und ihre Umweltkosten kennen. Jedoch zeigt eine bei 47 KMU verwirklichte Untersuchung, dass dieses Postulat nicht immer bestätigt werden kann, was die Wirksamkeit dieser Regulierungsinstrumente begrenzt. Sie hebt ebenfalls die Tatsache hervor, dass die Information keine ausreichende Bedingung ist für die Verbesserung der umweltorientierten Leistungen der Unternehmen. Einige Unternehmen profitieren bei ihren umweltorientierten Leistungen von der Informationsasymmetrie, indem sie ein opportunistisches Typenverhalten einnehmen. Dagegen wird gezeigt, dass mehrere Unternehmen eher Partnerschaften eingehen, wenn sie ihre unternehmensspezifische Umwelt integriert.

Revue internationale P.M.E., vol. 16, $\mathrm{n}^{\circ}$ 2, 2003 


\section{Introduction}

En France, l'industrie est responsable d'environ un tiers des émissions de $\mathrm{CO}_{2}$ et de $70 \%$ des émissions de dioxyde de soufre; elle consomme au moins $40 \%$ de l'eau distribuée par les réseaux publics ${ }^{1}$ et $30 \%$ des consommations énergétiques ${ }^{2}$. La part de responsabilité des PME/PMI dans la pollution industrielle globale est difficile à quantifier : il y a peu de données environnementales sur ces entreprises qui sont rarement soumises à l'autosurveillance et il est difficile d'agréger des informations relatives à des problématiques industrielles et environnementales hétérogènes. Cependant, on sait que les PME / PMI emploient plus de la moitié de la main-d'œuvre industrielle et fournissent près de la moitié du chiffre d'affaires de l'industrie manufacturière ${ }^{3}$. Si on fait l'hypothèse que la pollution est globalement proportionnelle à la production et à la main-d'œuvre, on peut grossièrement estimer que les PME/PMI sont responsables de près de la moitié de la pollution causée par l'industrie. Aussi, l'impact environnemental des PME / PMI préoccupe de plus en plus leurs parties intéressées (institutions, riverains, clients, fournisseurs, salariés, etc.). Cela se traduit, pour l'entreprise, par des enjeux réglementaires, économiques, stratégiques et managériaux croissants liés à l'intégration de l'environnement. Les mécanismes traditionnels de régulation, qui visent à inciter l'entreprise à internaliser ses impacts environnementaux, sont basés sur le postulat de l'information parfaite : l'approche réglementaire part du principe que «nul n'est censé ignorer la loi ». Pour assurer le bon fonctionnement des instruments économiques, l'entreprise doit effectuer un suivi de ses coûts environnementaux et trouver des solutions techniques lui permettant de réduire les prélèvements et rejets taxés. Les normes d'application volontaire ne sont utilisées que si les entreprises les connaissent et perçoivent leur intérêt.

Nous montrons dans cet article que ce postulat ne peut pas s'appliquer au cas de l'intégration de l'environnement par les PME. Dans un premier temps, nous définirons la notion «d'environnement » pour l'entreprise et présenterons les outils qui visent à réguler les externalités environnementales et leurs limites. Dans un second temps, nous présenterons les résultats d'une enquête que nous avons réalisée auprès de 47 chefs d'entreprise. Cette enquête montre les lacunes informationnelles des PME, leur tendance à adopter un comportement opportuniste et le rôle joué par leurs partenaires pour les encourager à réduire leurs impacts environnementaux.

1. Données IFEN (1996) disponibles sur Internet: <http://www.ifen.fr>.

2. Données du ministère de l'Industrie (2000): <http://www.industrie.gouv.fr/cgi-bin/ industrie/frame0.pl ?url=/energie/sommaire.htm>.

3. Données SESSI - Ministère français de l'Industrie : <http://www.minefi.gouv.fr/minefi/ chiffres/chiffres_industrie/index.htm>.

Revue internationale P.M.E., vol. 16, $\mathrm{n}^{\circ}$ 2, 2003

(C) 2003 - Presses de l'Université du Québec 


\section{Que signifie la notion d'environnement pour l'entreprise?}

La notion d'environnement prend différentes significations selon le cadre dans lequel on se place (sciences de la gestion ou écologie, par exemple). Pour l'entreprise, l'environnement peut être l'ensemble constitué, d'une part, des milieux dans lesquels elle prélève des ressources et rejette des résidus (air, eau, terre, ressources naturelles, flore, faune et êtres humains) et, d'autre part, des acteurs avec lesquels elle est en relation. Ces derniers lui envoient des informations et des exigences environnementales (plus ou moins explicites). Réciproquement, l'entreprise peut leur communiquer des informations sur ses impacts environnementaux, des garanties de bonne conduite, ou même imposer des exigences portant sur les questions environnementales à ses prestataires. Cette définition est illustrée à la figure 1.

FIGURE 1

\section{Représentation de l'environnement de l'entreprise}

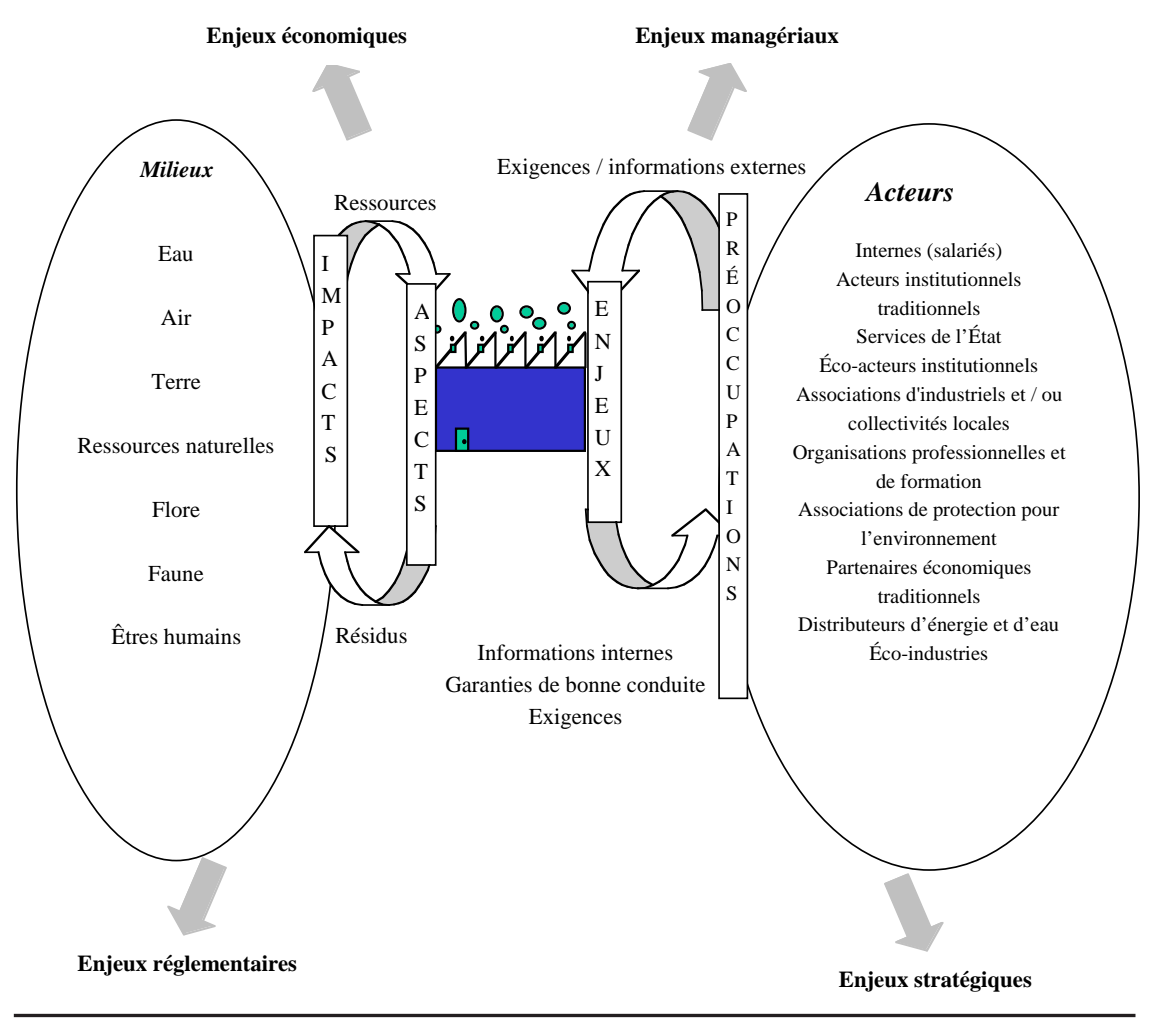

Revue internationale P.M.E., vol. 16, $\mathrm{n}^{\circ}$ 2, 2003 
Pour qualifier les relations entre l'entreprise et les milieux naturels, la norme ISO 14001 définit les termes aspect et impact environnemental comme étant, respectivement, un «élément des activités, produits ou services d'un organisme susceptible d'interagir avec l'environnement» et « toute modification de l'environnement, négative ou bénéfique, résultant totalement ou partiellement des activités, produits ou services d'un organisme» (ISO, 1996). Symétriquement pour les relations avec les acteurs, nous proposons les notions de préoccupations et d'enjeux environnementaux. Nous définissons un enjeu comme étant la « valeur que l'entreprise peut gagner ou perdre grâce à une amélioration de ses performances environnementales et de ses relations avec ses parties intéressées ${ }^{4} \gg$. Quatre types d'enjeux peuvent être distingués : réglementaires (éviter les risques juridiques et financiers liés à la non-conformité), économiques (maîtriser les coûts et assurer l'accès aux marchés), stratégiques (anticiper sur les évolutions ; Personne, 1998) et managériaux (motiver le personnel). Ces enjeux correspondent aux préoccupations des parties intéressées, c'est-à-dire aux aspects environnementaux de l'entreprise qui sont sujets d'intérêt ou d'inquiétude pour différents acteurs.

Pour une entreprise, intégrer l'environnement signifie donc, d'une part, réduire ses impacts sur le milieu naturel, ce qui lui permet de maîtriser les coûts liés à la valeur économique des ressources environnementales et les risques liés à sa responsabilité juridique en cas d'accident ou de pollution, et, d'autre part, améliorer ses relations avec ses parties intéressées, ce qui lui permet d'accroître sa valeur en termes d'éthique et d'image.

Trois types d'approches traditionnelles de régulation visent à inciter les entreprises à réduire leurs impacts sur l'environnement (Brodhag, 1994) :

- Dans l'approche régalienne, l'entreprise perçoit les informations et contraintes de l'environnement à travers la réglementation et les procédures administratives. Suivant cette approche, «nul n'est censé ignorer la loi » : les textes de loi ne sont appliqués qu'à condition que les réglementés les connaissent. Ces derniers doivent également savoir comment mesurer leurs impacts environnementaux pour évaluer leur degré de conformité par rapport aux référentiels réglementaires. Comme il est difficile, voire impossible, d'évaluer les dommages causés par la pollution, les réglementations sont élaborées par un processus politique visant à établir un consensus entre les parties prenantes. Cela produit souvent des textes complexes et ambigus (Faucheux et Noël, 1995), rendant difficile leur connaissance,

4. Une partie intéressée est un «individu ou groupe concerné ou affecté par la performance environnementale d'un organisme» (ISO, 1996).

Revue internationale P.M.E., vol. 16, $\mathrm{n}^{\circ}$ 2, 2003

(C) 2003 - Presses de l'Université du Québec 
compréhension et maîtrise par les industriels. De plus, l'évolution rapide $^{5}$ et difficilement prévisible de ces textes complique l'élaboration des stratégies à long terme des entreprises.

- Dans l'approche économique, utilisée parallèlement à l'approche réglementaire, l'entreprise perçoit les informations et contraintes sous forme financière (taxes, subventions ou marché des permis d'émission) selon le principe du «pollueur payeur ». L'objectif de ces outils est d'internaliser dans les charges de l'entreprise les coûts liés aux externalités environnementales négatives qu'elle engendre. D'une part, cette approche est limitée par les difficultés à évaluer économiquement les bénéfices de la dépollution (Faucheux et Noël, 1995). D'autre part, ces outils reposent sur l'hypothèse que l'entreprise connaît les coûts liés à la taxe, les économies qu'elle réalisera sur cette taxe si elle diminue ses impacts environnementaux et les solutions techniques qui lui permettent d'apporter cette amélioration.

- L'approche contractuelle donne la possibilité à l'entreprise d'entreprendre une démarche volontaire, allant au-delà de la réglementation, et de mettre en valeur cette démarche par une validation par une tierce partie. Les instruments de cette approche sont les négociations directes entre pollueurs et pollués, les engagements unilatéraux, les accords négociés ou contrats de branche (Börkey et Glachant, 1999) et les programmes volontaires (système de management environnemental ou écoproduits). Cette approche a la faveur des économistes libéraux (Faucheux et Noël, 1995). Cependant, son efficacité est limitée s'il n'y a pas de dispositif de contrôle et de sanction individuelle (Börkey et Glachant, 1999). De plus, ces outils ne sont utilisés que par les entreprises qui connaissent leur existence et les enjeux qui y sont liés.

Une forme de coordination moins traditionnelle et encore peu utilisée par les pouvoirs publics pour réguler les externalités environnementales est la coordination par les réseaux. Cette forme de coordination transversale permet aux acteurs de réduire de proche en proche l'incertitude et d'évaluer et orienter les actions de chaque membre (Aggeri, 1999). Le réseau est un moyen de diffusion des informations particulièrement adapté aux PME, car il favorise la confiance dans les relations entre l'entreprise et son interlocuteur (Julien, 1997). Les réseaux (associations d'industriels pour l'environnement, réseaux d'innovation, réseaux locaux rassemblant acteurs publics et privés, opérations collectives) favorisent la diffusion de techniques et pratiques moins polluantes et peuvent être considérés comme une

5. En 2000, près de 60 textes concernant la législation sur les installations classées pour l'environnement ont été publiés. 
forme de régulation des externalités environnementales en complément d'autres outils. Contrairement aux instruments économiques qui fournissent des contraintes fortes, mais aucune indication sur les moyens technologiques permettant une amélioration, les réseaux impliquent des contraintes faibles, mais donnent un cadre fort pour le progrès en facilitant les transferts de connaissances et de savoir-faire (Aggeri, 1999).

Ainsi, les outils traditionnels de régulation des externalités environnementales (outils réglementaires, économiques et contractuels) reposent sur l'hypothèse implicite que les entreprises possèdent suffisamment d'informations pour trouver les outils elles-mêmes et les solutions qu'elles peuvent appliquer pour avoir le comportement que l'on attend d'elles. Pourtant, ce postulat d'information parfaite peut être remis en cause, en particulier, dans le cas de l'intégration de l'environnement par les PME. En effet, d'une part, dans une PME, la décision repose souvent sur le seul chef d'entreprise et un système d'information simple et peu formalisé (Julien, 1997). Les capacités de traitement de l'information reposent donc sur les capacités humaines du chef d'entreprise et non sur l'organisation dans son ensemble. Cela amplifie le phénomène de rationalité limitée voulant qu'un être humain ne possède pas les capacités nécessaires au traitement exhaustif des informations qui lui seraient nécessaires pour prendre une décision en parfaite connaissance de cause (Simon, 1955).

D'autre part, l'information environnementale est caractérisée par sa complexité et son incertitude, ce qui rend impossible la connaissance a priori de l'évolution de l'univers de l'entreprise (Godard, 1993), même avec des capacités illimitées de traitement. L'environnement étant de surcroît considéré comme une contrainte secondaire et non comme un atout potentiel, le chef d'entreprise s'engage rarement dans une démarche active de recherche et de traitement de l'information environnementale. Aussi, la sous-information générale du chef d'entreprise et des employés des PME / PMI est déplorée par de nombreuses études (Hillary, 2000). Les chefs d'entreprise ont une faible perception des enjeux environnementaux (De Baker, 1999) et investissent peu de moyens dans la prévention de la pollution, s'enfermant ainsi dans un cercle de passivité (Gondran et Brodhag, 2000).

Ainsi, notre hypothèse est que la situation d'information imparfaite est amplifiée dans le cas des PME et de l'environnement. L'efficacité des outils traditionnels de régulation environnementale ne semble donc pas assurée.

Pour aider les entreprises et leurs parties intéressées à briser ce cercle de passivité, nous chercherons, par une enquête de terrain, à déterminer les canaux d'information environnementale les plus efficaces pour encourager les PME / PMI à améliorer leurs performances environnementales. 


\section{Une approche empirique pour observer le comportement environnemental des PME}

Notre démarche d'investigation est basée sur une enquête menée avec un questionnaire administré par entretien en face à face. Cette technique occasionne un coût plus élevé de collecte de données que l'envoi de questionnaire par voie postale, mais présente moins de risques de mauvaise interprétation des questions et donne lieu à de meilleurs taux de réponses (Quivy et Van Campenhoudt, 1995). Elle nous permet également d'éviter que :

- l'échantillon ne soit constitué que d'entreprises déjà avancées dans la prise en compte de l'environnement qui auraient été les seules à renvoyer le questionnaire pour communiquer sur l'environnement, notre objectif étant d'interroger des entreprises qui ne sont pas particulièrement sensibilisées au respect de l'environnement;

- les questions portant sur les connaissances du chef d'entreprise soient biaisées par le fait qu'il aurait eu le temps de faire des recherches avant de répondre afin de donner une meilleure image.

Les entretiens correspondaient à des prédiagnostics environnementaux, d'environ une heure, mettant en relation le niveau d'information environnementale auquel le chef d'entreprise a spontanément accès avec le niveau d'intégration de l'environnement de l'entreprise.

Les capacités d'accès à l'information sont mesurées à travers l'utilisation de supports écrits (presse spécialisée, Internet) et l'évaluation, par le chef d'entreprise, de l'influence accordée à divers partenaires sur sa politique générale puis sur les questions environnementales, sur une échelle de 0 (aucune relation) à 4 (partenaire influençant totalement l'entreprise).

Le niveau d'intégration de l'environnement est évalué à travers le niveau de connaissance environnementale du dirigeant ( $c f$. tableau 1 pour un exemple de question).

TABLEAU 1

Exemple de question évaluant

le niveau d'intégration de l'environnement par l'entreprise

Connaissez-vous la réglementation s'appliquant à ces rejets liquides?

$0 . \square$ NON

1. $\square$ OUI, mais elle n'est pas respectée.

2. $\square$ OUI, mais elle n'est pas toujours respectée.

3. $\square$ OUI, et les non-conformités sont surveillées.

4. $\square$ OUI, et il existe un plan de mise en conformité.

5. $\square$ OUI, et elle est respectée. 
Cette façon indirecte d'interroger les entreprises sur leur conformité à la réglementation permet d'accroître la confiance entre l'enquêteur et le répondant. Ainsi, ce dernier ne perçoit pas l'enquêteur comme un gendarme qui viendrait lui demander s'il est en conformité avec la réglementation. Pour avoir une vision globale du comportement environnemental de l'entreprise, nous avons utilisé les 15 critères présentés dans le tableau 4 ; ces critères ont été adaptés pour les PME à partir de ceux proposés par Butel-Belini (1997) grâce à des études de cas d'entreprises dans lesquelles nous avons réalisé des diagnostics environnementaux approfondis (Gondran et al., 2000). Cette grille permet de positionner chaque entreprise sur une échelle à trois niveaux en fonction de son comportement pour chaque critère. Le comportement écodéfensif correspond à une absence de prise en compte de l'environnement. Le niveau écoconformiste correspond au simple respect de la réglementation tandis que les entreprises écosensibles dépassent les exigences réglementaires et considèrent l'environnement comme un facteur de différenciation. Tous les critères ne sont pas applicables à toutes les PME (par exemple, certaines entreprises ne produisent pas de rejets liquides industriels). De plus, chaque entreprise peut avoir un comportement écodéfensif pour certains critères et écosensible pour d'autres. Ainsi, nous avons qualifié le niveau de comportement environnemental de chaque entreprise par trois ratios :

- \% crit-ecodef $=$ nombre de critères écodéfensifs / nombre de critères applicables à l'entreprise ;

- \% crit-ecoconf $=$ nombre de critères écoconformistes / nombre de critères applicables ;

- \% crit-ecosens $=$ nombre de critères écosensibles / nombre de critères applicables.

Pour vérifier la sincérité du répondant, plusieurs questions sont croisées afin de positionner l'entreprise selon chaque critère. Un programme, réalisé sur une feuille Excel ${ }^{\circledR}$, évalue le positionnement de chaque entreprise par rapport à chaque critère, puis ces trois ratios à partir des réponses au questionnaire.

Tous les entretiens ont été réalisés avec le même questionnaire mais par des organismes différents et dans différentes régions. Cela nous a permis, d'une part, de comparer les comportements des entreprises entre différents territoires et secteurs d'activité français et, d'autre part, de valider, auprès de différents acteurs et contextes, cette nouvelle méthode rapide d'évaluation environnementale des entreprises (Gondran, 2001). Pour valider cette méthode et obtenir un premier échantillon d'entreprises, nous avons employé la démarche décrite sur la figure 2.

Revue internationale P.M.E., vol. 16, $\mathrm{n}^{\circ}$ 2, 2003

(C) 2003 - Presses de l'Université du Québec 
FiguRE 2

Méthode d'investigation suivie pour l'enquête par questionnaire

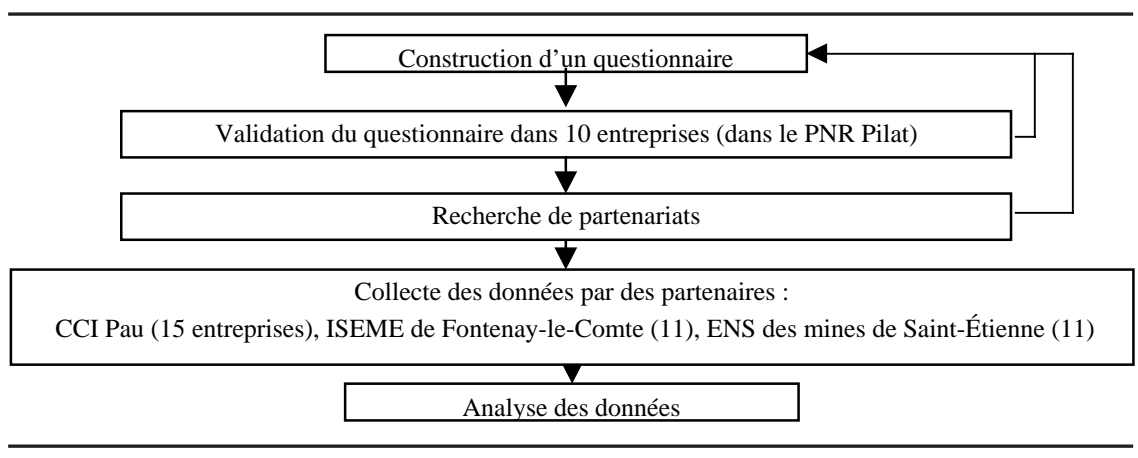

Ainsi, le questionnaire devait pouvoir être utilisé dans différents contextes et à différentes fins (sensibiliser des industriels à l'environnement, améliorer la connaissance du tissu industriel local ou amener des étudiants à mieux connaître le milieu industriel). Les biais liés au statut de l'enquêteur introduits par cette démarche sont limités par le caractère fermé des questions à choix multiples exempts d'ambiguïté.

Chaque organisme nous a renvoyé les questionnaires remplis pour une dizaine d'entreprises. Notre échantillon est ainsi composé de trois sous-échantillons représentant trois régions françaises (tableau 2). Les entretiens ont été effectués entre juillet 1999 (validation dans les 10 premières entreprises) et décembre 2000. Le

TABleau 2

Caractéristiques géographiques de l'échantillon

\begin{tabular}{cccc}
\hline Lieu & Structure porteuse & $\begin{array}{c}\text { Entreprises } \\
\text { concernées } \\
\text { interrogées }\end{array}$ & $\begin{array}{c}\text { Nombre } \\
\text { d'entreprises }\end{array}$ \\
\hline $\begin{array}{c}\text { Fontenay-le-Comte* } \\
\text { (Vendée) }\end{array}$ & $\begin{array}{c}\text { Un consultant encadrant } \\
\text { 6 élèves de BTS environnement }\end{array}$ & $\begin{array}{c}\text { Club de } \\
60 \text { entrepreneurs }\end{array}$ & 11 \\
\hline $\begin{array}{c}\text { Pau** } \\
\text { (Pyrénées-Atlantiques) }\end{array}$ & CCI de Pau & Ressortissants & 15 \\
\hline Rhône-Alpes & ENS des mines & $\begin{array}{c}\text { PNR du Pilat } \\
+ \text { Loire*** }\end{array}$ & 10 \\
& & +11 \\
\hline
\end{tabular}

* Avec la collaboration de Michel Lagoutte, consultant environnement, 4, rue Belgrand, 75020 Paris, pour l'ISEME (Institut supérieur des métiers de l'environnement).

** Avec la collaboration de Patricia Roques, CCI de Pau, 21, rue Louis-Barthou, B.P. 128, $64001 \mathrm{Pau}$.

*** Avec la participation de Frédéric Poulard, élève de troisième année de l'ENS des mines de Saint-Étienne et de Denis Chazallet, de la CCI de Saint-Étienne / Montbrison. 
nombre total d'entreprises interrogées (47) est trop faible pour être statistiquement représentatif de l'ensemble des secteurs géographiques et d'activité français, mais il ouvre des pistes de recherche pour une étude exploratoire dans un domaine (les PME et l'environnement) sur lequel la littérature scientifique est peu abondante.

Chaque partenaire s'est efforcé de constituer un échantillon représentatif de sa région en termes de secteurs industriels (textile, travail des métaux, mécanique, bois, caoutchouc et plastique, agroalimentaire). Le tableau 3 montre que notre échantillon est fidèle à la répartition des PME / PMI pour ce qui est de la taille.

TABLEAU 3

Taille des entreprises interrogées

\begin{tabular}{lcc}
\hline & Échantillon total & $\begin{array}{c}\text { Pourcentage parmi les entreprises } \\
\text { répertoriées dans le Kompass }\end{array}$ \\
\hline Petites (1 à 49) & $81 \%$ & $80 \%$ \\
Moyennes (50 à 249) & $19 \%$ & $20 \%$ \\
ENSEMBLE & $100 \%$ (47 sites) & $100 \%(113800$ sites $)$ \\
\hline
\end{tabular}

\section{La prise en compte de l'environnement et l'ouverture sur des partenaires}

Le tableau 4 présente un tri à plat des critères choisis pour évaluer les performances environnementales des entreprises : le pourcentage représente le nombre d'entreprises classées au niveau correspondant à la colonne par rapport au nombre d'entreprises concernées par le critère.

Pour 9 critères sur 15, la majorité des PME ont un comportement écodéfensif. Parmi ces critères, six concernent les pratiques opérationnelles de l'entreprise en termes de maîtrise de la pollution (aucune gestion des consommations en eau et énergie).

Pour cinq critères, les PME ont majoritairement un comportement écoconformiste. Ces critères montrent que les entreprises ont reçu une première sensibilisation à la nécessité d'intégrer l'environnement (demande des clients, sensibilisation du personnel).

Le seul critère pour lequel $60 \%$ des entreprises interrogées peuvent être qualifiées d'écosensibles est leur perception d'enjeux environnementaux stratégiques. Ce chiffre est probablement surestimé par rapport à la population totale des entreprises françaises étant donné l'absence d'obligation de la part des

Revue internationale P.M.E., vol. 16, $\mathrm{n}^{\circ}$ 2, 2003 
TABleau 4

Pourcentage d'entreprises (sur les 47 sondées) possédant les caractéristiques d'un comportement écodéfensif, écoconformiste ou écosensible

\begin{tabular}{|c|c|c|c|}
\hline Action & Écodéfensif & Écoconformiste & Écosensible \\
\hline Responsable environnement & $\begin{array}{l}\text { Aucun } \\
60 \%\end{array}$ & $\begin{array}{c}\text { Cadre à temps partiel } \\
40 \%\end{array}$ & $\begin{array}{c}\text { Service formalisé } \\
0 \%\end{array}$ \\
\hline Connaissance de la réglementation & Aucune & $\begin{array}{c}\text { Identification des } \\
\text { principaux textes } \\
\text { s'appliquant à l'activité, } \\
\text { sans veille } \\
55 \%\end{array}$ & $\begin{array}{c}\text { Veille réglementaire } \\
\text { environnementale }\end{array}$ \\
\hline $\begin{array}{l}\text { Respect de la législation pour les rejets } \\
\text { liquides (pour } 30 \text { entreprises } \\
\text { concernées) }\end{array}$ & $\begin{array}{l}\text { Non-conformité } \\
60 \% \\
\end{array}$ & $\begin{array}{c}\text { Conformité partielle, } \\
\text { non-conformités connues } \\
10 \%\end{array}$ & $\begin{array}{l}\text { Conformité totale } \\
30 \% \\
\end{array}$ \\
\hline $\begin{array}{l}\text { Respect de la législation pour les rejets } \\
\text { atmosphériques (pour } 21 \text { entreprises } \\
\text { concernées) }\end{array}$ & $\begin{array}{l}\text { Non-conformité } \\
62 \% \\
\end{array}$ & $\begin{array}{c}\text { Conformité partielle, } \\
\text { non-conformités connues } \\
10 \% \\
\end{array}$ & $\begin{array}{c}\text { Conformité } \\
28 \% \\
\end{array}$ \\
\hline Gestion des consommations en eau & $\begin{array}{c}\text { Aucune gestion } \\
46 \% \\
\end{array}$ & $\begin{array}{c}\text { Contrôle des } \\
\text { consommations } \\
\text { sans optimisation } \\
43 \% \\
\end{array}$ & $\begin{array}{c}\text { Optimisation } \\
\text { des consommations } \\
\text { et suivi formalisé } \\
11 \% \\
\end{array}$ \\
\hline $\begin{array}{l}\text { Gestion des consommations } \\
\text { énergétiques }\end{array}$ & Aucune gestion & $\begin{array}{c}\text { Quelques actions } \\
\text { dispersées } \\
22 \%\end{array}$ & $\begin{array}{c}\text { Optimisation } \\
\text { des consommations } \\
\text { et suivi formalisé } \\
36 \%\end{array}$ \\
\hline $\begin{array}{l}\text { Intégration de l'environnement dans la } \\
\text { gestion des déchets d'emballage }\end{array}$ & $\begin{array}{l}\text { Aucune } \\
50 \%\end{array}$ & $\begin{array}{l}\text { Valorisation interne } \\
\text { ou externe } \\
37 \%\end{array}$ & $\begin{array}{c}\text { Réduction } \\
\text { à la source } \\
13 \%\end{array}$ \\
\hline
\end{tabular}

entreprises de participer à l'enquête: les entreprises n'ayant aucune conscience environnementale refusaient généralement l'entretien ${ }^{6}$.

Il semble donc que les entreprises aient une conscience diffuse des enjeux stratégiques liés à l'environnement, mais qu'elles n'aient pas intégré cette dimension à leur propre stratégie. La question qui se pose alors est de savoir si les entreprises ont accès à suffisamment d'information pour intégrer ces enjeux et avoir le comportement que les instances régulatrices attendent d'elles en réponse aux instruments réglementaires, économiques et volontaires qui sont mis en place pour réguler les externalités environnementales.

Grâce aux entretiens réalisés au cours de l'enquête et à l'analyse des renseignements fournis à des entreprises par des associations d'industriels (Gondran, 2001), nous proposons la typologie suivante des informations environnementales nécessaires aux entreprises:

6. Le taux d'acceptation de l'entretien variait entre $19 \%$ et $50 \%$ selon les partenaires chargés de l'enquête. 
TABLEAU 4 (suite)

\begin{tabular}{|c|c|c|c|}
\hline \multicolumn{4}{|c|}{$\begin{array}{l}\text { Pourcentage d'entreprises (sur les } 47 \text { sondées) possédant les caractéristiques } \\
\text { d'un comportement écodéfensif, écoconformiste ou écosensible }\end{array}$} \\
\hline Action & Écodéfensif & Écoconformiste & Écosensible \\
\hline Terme de plan d'action & $\begin{array}{l}\text { Au coup } \\
\text { par coup } \\
28 \%\end{array}$ & $\begin{array}{l}\text { À court terme } \\
\text { (un an) } \\
36 \%\end{array}$ & $\begin{array}{l}\text { Après planification } \\
\text { à MT, voire LT } \\
36 \%\end{array}$ \\
\hline Réalisation d'études d'impact & $\begin{array}{l}\text { Aucune } \\
\text { ou nsp } \\
57 \%\end{array}$ & $\begin{array}{l}\text { Études imposées } \\
\quad \text { par la loi } \\
\quad 34 \%\end{array}$ & $\begin{array}{l}\text { Études } \\
\text { complémentaires } \\
9 \%\end{array}$ \\
\hline $\begin{array}{l}\text { Perception des investissements } \\
\text { écologiques }\end{array}$ & $\begin{array}{l}\text { Coût } \\
\text { à éviter } \\
4 \%\end{array}$ & $\begin{array}{l}\text { Coût obligatoire } \\
\text { à minimiser } \\
68 \%\end{array}$ & $\begin{array}{l}\text { Investissement } \\
\text { rentable } \\
28 \% *\end{array}$ \\
\hline $\begin{array}{l}\text { Relations environnementales avec } \\
\text { les fournisseurs }\end{array}$ & $\begin{array}{l}\text { Aucune } \\
51 \%\end{array}$ & $\begin{array}{c}\text { Conseils techniques } \\
34 \%\end{array}$ & $\begin{array}{c}\text { Collaboration poussée } \\
15 \%\end{array}$ \\
\hline Relations avec le marché & $\begin{array}{l}\text { Aucune exigence } \\
\text { client } \\
57 \%\end{array}$ & $\begin{array}{c}\text { Préoccupation } \\
\text { des clients } \\
40 \% \\
\end{array}$ & $\begin{array}{l}\text { En plus : opportunité } \\
\text { d'activité } \\
3 \%\end{array}$ \\
\hline $\begin{array}{l}\text { Sensibilisation du personnel aux } \\
\text { risques liés aux produits dangereux } \\
\text { (pour } 33 \text { entreprises concernées) }\end{array}$ & $\begin{array}{c}\text { Aucune } \\
24 \% \\
\end{array}$ & $\begin{array}{l}\text { Sensibilisation (affichage, } \\
\text { conseils informels) } \\
76 \%\end{array}$ & $\begin{array}{c}\text { Formation et consignes } \\
\text { de sécurité formalisées } \\
0 \%\end{array}$ \\
\hline $\begin{array}{l}\text { Flux d'information environnementale } \\
\text { à l'interne }\end{array}$ & $\begin{array}{c}\text { Inexistant } \\
41 \%\end{array}$ & $\begin{array}{c}\text { Actions } \\
\text { de sensibilisation } \\
38 \%\end{array}$ & $\begin{array}{c}\text { Actions organisées } \\
\text { de responsabilisation } \\
21 \%\end{array}$ \\
\hline $\begin{array}{l}\text { Perception des enjeux stratégiques } \\
\text { liés à l'environnement }\end{array}$ & $\begin{array}{l}\text { Aucune } \\
\text { perception } \\
\text { d'enjeux } \\
\text { stratégiques }\end{array}$ & $\begin{array}{l}\text { Recherche d'amélioration } \\
\text { des relations avec les } \\
\text { parties intéressées, de } \\
\text { diminution des coûts ou } \\
\text { enjeux managériaux sans } \\
\text { enjeux liés au marché } \\
29 \%\end{array}$ & $\begin{array}{c}\text { Perception d'enjeux } \\
\text { liés } \\
\text { au marché } \\
\text { ou à la } \\
\text { pérennité } \\
\text { de l'entreprise } \\
60 \%\end{array}$ \\
\hline
\end{tabular}

* Par exemple, une scierie a investi dans une chaufferie au bois pour brûler ces sciures et se chauffer l'hiver, ce qui lui permet de ne plus utiliser de combustible fossile.

- Les informations sur l'environnement, au sens de la Convention de Aarhus portent sur «l'état d'éléments de l'environnement et l'interaction entre ces éléments; des facteurs qui ont des incidences sur l'environnement, activités ou mesures, y compris mesures administratives, des accords relatifs à l'environnement, des politiques, plans et programmes qui ont ou risquent d'avoir des incidences sur les éléments de l'environnement et l'analyse coût-avantage et les autres analyses et hypothèses économiques utilisées dans les processus décisionnels en matière d'environnement ; l'état de santé de l'homme, sa sécurité et ses conditions de vie ainsi que l'état des sites culturels et des constructions dans la mesure où ils sont ou risque d'être affectés, par l'état des éléments de l'environnement » (CENUE, 1998). 
- Les informations sur les acteurs de l'environnement (institutionnels, économiques et organisations collectives) qui peuvent assister l'entreprise dans sa démarche environnementale aident l'entreprise à choisir ses partenaires.

- Les informations sur les moyens permettent à l'entreprise de connaître les solutions qui sont à sa disposition pour diminuer ses impacts : biens et équipements nécessaires, matières premières et consommables, méthodes et connaissances mobilisables.

- Les informations sur les enjeux liés à l'intégration de l'environnement par l'entreprise sont des arguments montrant à l'entreprise ce qu'elle peut gagner en intégrant mieux l'environnement.

- Les informations sur les événements et références permettant aux entreprises de savoir où et quand communiquer et s'informer.

L'analyse de 315 renseignements fournis à des entreprises par deux centres de ressources révèle que les deux types d'information les plus recherchés par les industriels sont les informations sur l'environnement (en particulier, les mesures administratives), représentant $56 \%$ des renseignements fournis, et l'information sur les acteurs (29\%; Gondran, 2001). Dans la première section, nous avons vu que les outils traditionnels de régulation postulaient la situation d'information parfaite des entreprises et nous avons mis en évidence les principaux types d'informations nécessaires aux entreprises pour assurer l'efficacité de ces outils. Les différents types d'information nécessaires sont repris selon cette typologie dans le tableau 5.

Afin de respecter les contraintes de temps des entrepreneurs, nous avons sélectionné, en collaboration avec les partenaires qui ont utilisé le questionnaire, les types d'informations les plus significatifs pour les entreprises. Les modalités de la fiscalité environnementale ayant évolué au cours de l'enquête (introduction de la taxe généralisée sur les activités polluantes), nos partenaires se sont montrés réticents pour poser des questions à ce propos et nous n'avons donc pas interrogé les entreprises sur leurs connaissances des outils fiscaux.

Soixante pour cent des entreprises ne possèdent pas de responsable environnement et les chefs d'entreprise assurent, en plus de leurs fonctions traditionnelles, la fonction «environnement». Quarante pour cent ont un responsable environnement cumulant d'autres responsabilités (qualité, sécurité, maintenance). Aucun responsable environnement à temps plein n'a été nommé. Aussi, les capacités de recherche et de traitement de l'information environnementale des PME sont limitées. Le tableau 6 donne la répartition des entreprises interrogées selon leur niveau d'accès aux informations sélectionnées. 
TABLEAU 5

Informations requises pour l'utilisation du postulat d'information parfaite

\begin{tabular}{|c|c|c|c|}
\hline & $\begin{array}{l}\text { Approche } \\
\text { réglementaire }\end{array}$ & $\begin{array}{l}\text { Approche } \\
\text { économique }\end{array}$ & Approche contractuelle \\
\hline $\begin{array}{l}\text { Informations } \\
\text { sur } \\
\text { l'environnement }\end{array}$ & $\begin{array}{l}\text { Textes de loi et leur } \\
\text { interprétation. }\end{array}$ & Outils fiscaux. & $\begin{array}{c}\text { Sensibilité de } \\
\text { l'environnement local. }\end{array}$ \\
\hline \multicolumn{4}{|c|}{ Nature des impacts environnementaux de l'entreprise } \\
\hline $\begin{array}{l}\text { Information sur } \\
\text { les acteurs }\end{array}$ & Instances de contrôle. & $\begin{array}{c}\text { Instances chargées } \\
\text { de prélever les taxes. }\end{array}$ & $\begin{array}{l}\text { Organismes proposant } \\
\text { des référentiels. }\end{array}$ \\
\hline $\begin{array}{l}\text { Informations } \\
\text { sur les moyens }\end{array}$ & $\begin{array}{l}\text { Moyens de mesure des } \\
\text { impacts industriels. }\end{array}$ & $\begin{array}{l}\text { Solutions permettant } \\
\text { de réduire les } \\
\text { consommations et les } \\
\text { rejets. }\end{array}$ & $\begin{array}{l}\text { Méthodes pour satisfaire } \\
\text { les exigences de } \\
\text { l'engagement contractuel, } \\
\text { les bonnes pratiques. }\end{array}$ \\
\hline $\begin{array}{l}\text { Informations } \\
\text { sur les enjeux }\end{array}$ & $\begin{array}{l}\text { Conséquences des non- } \\
\text { conformités } \\
\text { (responsabilité du } \\
\text { dirigeant, amendes). }\end{array}$ & $\begin{array}{c}\text { Connaissance des } \\
\text { coûts liés aux taxes et } \\
\text { de leur évolution. }\end{array}$ & $\begin{array}{c}\text { Enjeux stratégiques liés } \\
\text { à l'environnement } \\
\text { pour le secteur } \\
\text { d'activité. }\end{array}$ \\
\hline $\begin{array}{l}\text { Informations } \\
\text { sur les } \\
\text { événements et } \\
\text { références }\end{array}$ & $\begin{array}{l}\text { Journées d'informations } \\
\text { sur la réglementation } \\
\text { et son évolution. } \\
\text { Bibliographie } \\
\text { sur le droit de } \\
\text { l'environnement. }\end{array}$ & $\begin{array}{c}\text { Journées } \\
\text { d'informations } \\
\text { sur la fiscalité } \\
\text { et son évolution. }\end{array}$ & $\begin{array}{c}\text { Journées d'informations sur } \\
\text { les normes (management } \\
\text { environnemental). } \\
\text { Bibliographie } \\
\text { et référentiels de } \\
\text { management environnemental. }\end{array}$ \\
\hline
\end{tabular}

Pour 8 catégories d'information sur les 19 choisies, un manque d'information est constaté au sein de l'entreprise : $25 \%$ (pour les rejets liquides) à $55 \%$ (pour les déchets) des PME interrogées ignorent les textes de loi auxquels elles sont soumises ; $12 \%$ (pour le bruit) à $67 \%$ (pour les émissions atmosphériques) des entreprises manquent d'informations sur les conséquences sur l'environnement de leurs procédés; $40 \%$ à $60 \%$ des PME ne connaissent pas les comportements alternatifs (solutions techniques et organisationnelles) qu'elles pourraient adopter pour internaliser leurs impacts environnementaux.

La plupart des PME n'ont donc pas accès aux informations nécessaires pour modifier leur comportement en fonction des instruments de régulation des externalités environnementales que ceux-ci soient réglementaires, économiques ou contractuels. Cela valide notre hypothèse : le postulat d'information parfaite sur lequel se fondent les outils traditionnels de régulation des externalités environnementales ne peut s'appliquer au cas des PME et de l'intégration de l'environnement.

Pour pouvoir appliquer ces modalités de régulation, les entreprises font part d'un besoin de simplification, de lisibilité et de clarification de ce que signifient 
TABLEAU 6

Niveau d'information des entreprises

\begin{tabular}{|c|c|c|c|c|}
\hline Thème environnemental & $\begin{array}{l}\text { Nombre } \\
\text { d'entreprises } \\
\text { concernées }\end{array}$ & $\begin{array}{l}\text { Un manque } \\
\text { d'information } \\
\text { est constaté. }\end{array}$ & $\begin{array}{l}\text { Pense être } \\
\text { suffisamment } \\
\text { informé. }\end{array}$ & $\begin{array}{l}\text { Principales sources } \\
\text { d'information relevées }\end{array}$ \\
\hline \multicolumn{5}{|c|}{ Nature des impacts environnementaux de l'entreprise } \\
\hline Nature des rejets liquides & 30 & $50 \%$ & $50 \%$ & \\
\hline $\begin{array}{l}\text { Nature des rejets } \\
\text { atmosphériques }\end{array}$ & 31 & $32 \%$ & $68 \%$ & $\begin{array}{l}\text { Médecin du travail, CRAM, } \\
\text { fournisseurs, CCI, laboratoire } \\
\text { agréé. }\end{array}$ \\
\hline Mesures de bruit & 34 & $12 \%$ & $88 \%$ & $\begin{array}{l}\text { Médecin du travail, CRAM, } \\
\text { voisinage. }\end{array}$ \\
\hline $\begin{array}{l}\text { Impacts des émissions } \\
\text { atmosphériques sur les } \\
\text { employés }\end{array}$ & 31 & $52 \%$ & $48 \%$ & $\begin{array}{l}\text { Médecin du travail, CRAM, } \\
\text { fournisseurs. }\end{array}$ \\
\hline $\begin{array}{l}\text { Impacts des émissions } \\
\text { atmosphériques sur la } \\
\text { population }\end{array}$ & 21 & $67 \%$ & $33 \%$ & $\begin{array}{l}\text { Médecin du travail, CRAM, } \\
\text { EDF, voisins. }\end{array}$ \\
\hline $\begin{array}{l}\text { Risques liés à } \\
\text { l'élimination des déchets }\end{array}$ & 33 & $42 \%$ & $58 \%$ & $\begin{array}{l}\text { Fournisseurs (FDS), CRAM, } \\
\text { DRIRE, bureau d'études. }\end{array}$ \\
\hline \multicolumn{5}{|c|}{ Approche réglementaire } \\
\hline $\begin{array}{l}\text { Réglementation } \\
\text { sur les déchets }\end{array}$ & 47 & $55 \%$ & $46 \%$ & \\
\hline $\begin{array}{l}\text { Réglementation sur les } \\
\text { rejets liquides }\end{array}$ & 32 & $27 \%$ & $73 \%$ & \\
\hline $\begin{array}{l}\text { Réglementation sur les } \\
\text { émissions atmosphériques }\end{array}$ & 21 & $38 \%$ & $62 \%$ & \\
\hline $\begin{array}{l}\text { Réglementation } \\
\text { sur le bruit }\end{array}$ & 42 & $31 \%$ & $69 \%$ & \\
\hline $\begin{array}{l}\text { Moyens d'analyse } \\
\text { des fumées }\end{array}$ & 21 & $52 \%$ & $48 \%$ & CRAM, médecin du travail. \\
\hline $\begin{array}{l}\text { Moyens de traitement des } \\
\text { émissions atmosphériques }\end{array}$ & 30 & $40 \%$ & $60 \%$ & $\begin{array}{l}\text { Médecin du travail, CRAM, } \\
\text { fournisseurs. }\end{array}$ \\
\hline $\begin{array}{l}\text { Relation avec les } \\
\text { instances de contrôle }\end{array}$ & 47 & $68 \%$ & $32 \%$ & DRIRE, DDAS. \\
\hline \multicolumn{5}{|c|}{ Approche économique } \\
\hline Consommations en eau & 47 & $46 \%$ & $54 \%$ & Factures d'eau. \\
\hline $\begin{array}{l}\text { Diminution des } \\
\text { consommations en eau }\end{array}$ & 47 & $67 \%$ & $33 \%$ & \\
\hline $\begin{array}{l}\text { Technologies sobres } \\
\text { en énergie }\end{array}$ & 42 & $60 \%$ & $40 \%$ & $\begin{array}{l}\text { EDF / GDF, fournisseurs, } \\
\text { ADEME. }\end{array}$ \\
\hline \multicolumn{5}{|c|}{ Approche contractuelle } \\
\hline $\begin{array}{l}\text { Sensibilité de } \\
\text { l'environnement local }\end{array}$ & 39 & $46 \%$ & $54 \%$ & $\begin{array}{l}\text { Étude d'impact, permis de } \\
\text { construire, bureau d'études, } \\
\text { collectivité locale. }\end{array}$ \\
\hline $\begin{array}{l}\text { Moyens de réduction } \\
\text { à la source des émissions } \\
\text { atmosphériques }\end{array}$ & 27 & $52 \%$ & $48 \%$ & $\begin{array}{l}\text { Médecin du travail, } \\
\text { fournisseurs, CCI, CRAM, } \\
\text { syndicat professionnel. }\end{array}$ \\
\hline Enjeux stratégiques & 47 & $31 \%$ & $69 \%$ & \\
\hline
\end{tabular}

Revue internationale P.M.E., vol. 16, $\mathrm{n}^{\circ}$ 2, 2003 
les textes pour leur cas particulier. Les comportements que ces outils souhaitent induire doivent donc être définis plus explicitement et les moyens que les entreprises peuvent prendre pour les manifester doivent être diffusés plus largement.

La question qui découle de cette hypothèse est de savoir si l'accès à l'information est une condition suffisante à l'amélioration des performances environnementales des PME.

FIGURE 3

Tri à plat sur les questions portant sur la connaissance et le respect des textes réglementaires

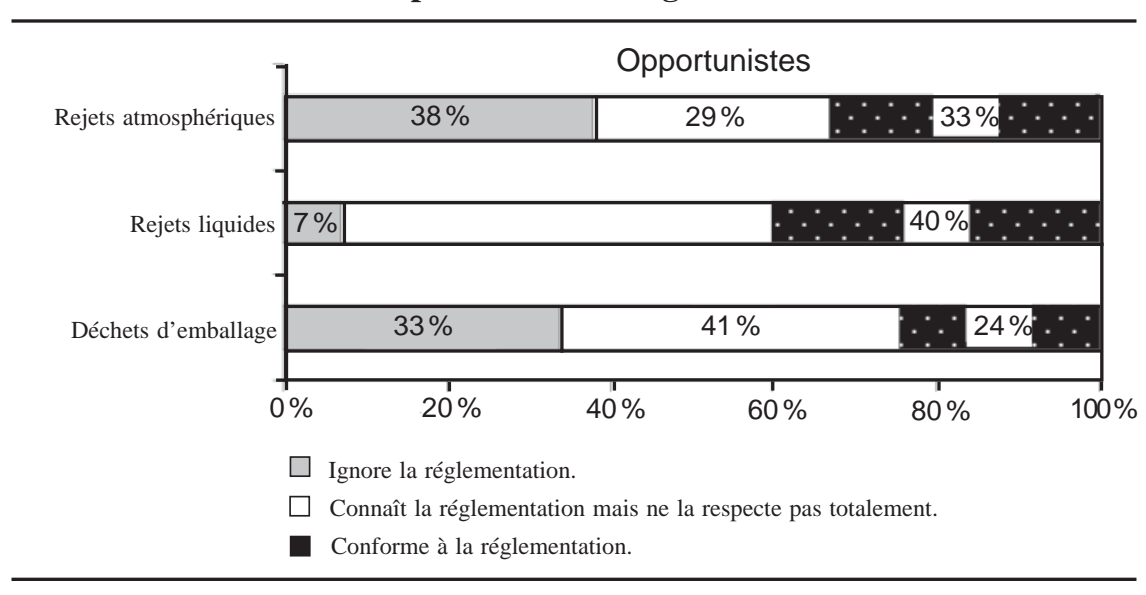

Cette figure montre qu'une proportion non négligeable d'entreprises avouent connaitre la réglementation mais ne pas la respecter totalement: pour les rejets liquides jusqu'à $53 \%$ des entreprises interrogées sont dans ce cas. Ce chiffre met en évidence le comportement opportuniste des PME / PMI, c'est-à-dire leur disposition, «pour réaliser leur intérêt personnel, à utiliser la ruse ou la tricherie ou à épuiser, de bonne foi, toutes les possibilités d'interprétation des contrats» (Koenig, 1993). Cet opportunisme est lié à l'asymétrie d'information (Reynaud, 1997), qui est due, notamment, à un manque de moyens des instances de contrôle ${ }^{7}$, à l'absence d'obligation de transparence ${ }^{8}$ par rapport aux émissions de la majorité

7. Il y a en moyenne un inspecteur DRIRE pour 86 installations soumises à autorisation (données provenant du site : <http://www.drire.gouv.fr/>) et 674 entreprises soumises à déclaration.

8. Depuis 1996, la législation nord-américaine impose aux industries les plus polluantes de communiquer leurs émissions polluantes (http://www.epa.gov/tri/) au grand public. Cette obligation de communication sur les performances environnementales est entrée dans la législation française en 2001 avec la Loi du 15 mai 2001 sur les nouvelles

Revue internationale P.M.E., vol. 16, $\mathrm{n}^{\circ}$ 2, 2003 
des PME, à l'ambiguïté des textes de loi qui donnent lieu à plusieurs interprétations possibles, aux difficultés internes des PME pour évaluer leurs propres performances ainsi qu'au manque d'expertise environnementale de certaines parties intéressées (municipalités, consommateurs, etc.).

Ainsi, il semble nécessaire de repérer les canaux d'information les plus efficaces pour inciter les PME à améliorer leurs performances environnementales. Pour ce faire, nous avons demandé aux chefs d'entreprise de noter, sur une échelle de 0 (aucune relation) à 4 (influence totale), l'influence qu'ils accordent à différents partenaires sur leurs politiques générales puis environnementales. Les partenaires dont l'influence a été ainsi évaluée ont été sélectionnés par recherche bibliographique et grâce aux premiers entretiens de l'échantillon de validation.

L'observation des moyennes des notes accordées aux différents partenaires (tableau 7) indique que les acteurs qui ont le plus d'influence sur la PME sont ceux avec lesquels elle est en contact direct: clients, médecin du travail, fournisseurs, Parc naturel régional pour la politique générale et, pour les questions liées à l'environnement, Parc naturel régional, CRAM (Caisse régionale d'assurance maladie), médecin du travail et clients.

L'étude des corrélations entre les notes données par les entreprises sur leur politique générale et celles portant sur leur gestion de l'environnement met en évidence trois types de partenaires :

- Ceux dont l'indice de corrélation est faible: il s'agit des partenaires économiques traditionnels (clients, fournisseurs, banques) qui n'envoient généralement aucun signal portant sur l'environnement.

- Ceux dont l'indice de corrélation est proche de 1. Il s'agit des interlocuteurs privilégiés pour les questions liées à environnement: population voisine, Agence de l'eau, bureau d'études et CCI.

- Ceux dont le coefficient de corrélation est faible, mais dont l'influence est plus forte par rapport à l'environnement que par rapport à la politique générale de l'entreprise $\left(R_{e / g}\right.$ supérieur à 1$)$ : le seul partenaire dans cette situation est le Parc naturel régional, interlocuteur surtout considéré pour son rôle concernant les questions liées à l'environnement.

régulations économiques qui ne concerne pour l'instant que les entreprises cotées en Bourse. Cependant, la Communication du 2 juillet 2002 de la Commission européenne concernant la responsabilité sociale des entreprises encourage explicitement l'engagement des PME dans de telles démarches. 
TABLEAU 7

Corrélations entre les influences sur la politique générale et sur la politique environnementale

\begin{tabular}{lcccc}
\hline & $\mathbf{M}_{\mathbf{g}}{ }^{\mathbf{a}}$ & $\mathbf{M}_{\mathbf{e}}^{\mathbf{b}}$ & Corrélation $^{\mathbf{c}}$ & $\mathbf{R}_{\mathbf{e} / \mathbf{g}} \mathbf{d}^{\mathbf{d}}$ \\
\hline Clients & 3,2 & 1,8 & 0,41 & 0,56 \\
Fournisseurs & 2,2 & 1,4 & 0,21 & 0,55 \\
Centre technique industriel & 1,7 & 1,5 & 0,77 & 0,82 \\
CCI & $\mathbf{1 , 7}$ & $\mathbf{1 , 5}$ & $\mathbf{0 , 8 3}$ & $\mathbf{0 , 9 1}$ \\
CRAM & & 2,0 & & \\
Médecins du travail & 2,5 & 1,9 & 0,74 & 0,75 \\
Inspecteurs du travail & 1,5 & 1,0 & 0,82 & 0,67 \\
DRIRE & 1,3 & 1,1 & 0,79 & 0,79 \\
Municipalité & $\mathbf{1 , 3}$ & $\mathbf{1 , 0}$ & $\mathbf{0 , 8 3}$ & 0,75 \\
Agence de l'eau & $\mathbf{0 , 8}$ & $\mathbf{0 , 9}$ & $\mathbf{0 , 8 9}$ & $\mathbf{1 , 0 0}$ \\
Compagnie d'assurances & 1,6 & 1,0 & 0,68 & 0,63 \\
Banque & 1,4 & 0,5 & 0,43 & 0,36 \\
Population voisine & 0,8 & 0,8 & 0,94 & 1,00 \\
Entreprises de même activité & 1,1 & 0,8 & 0,72 & 0,80 \\
Entreprises voisines & 0,7 & 0,6 & 0,80 & 0,83 \\
Bureau d'études & $\mathbf{1 , 0}$ & $\mathbf{1 , 0}$ & $\mathbf{0 , 8 0}$ & $\mathbf{1 , 0 0}$ \\
Parc naturel régional & 2,0 & 2,4 & 0,79 & $\mathbf{1 , 2 0}$ \\
\hline
\end{tabular}

a $\mathrm{M}_{\mathrm{g}}$ : moyenne de la note «influence générale du partenaire sur l'entreprise».

b $\mathrm{M}_{\mathrm{e}}$ : moyenne de la note «influence par rapport à l'environnement».

c Corrélation entre les notes données par les entreprises pour l'influence sur la politique générale et celles données par rapport à l'environnement.

${ }^{d} R_{e / g}=M_{e} / M_{g}$

e L'influence de la CRAM est évaluée par rapport aux aspects « risques industriels » seulement.

Les écarts types de ces notes sont très élevés (les coefficients de variation sont souvent proches de 1). Il n'y a donc pas de partenaire unanimement reconnu comme jouant un rôle primordial sur la politique de toutes les PME, mais chaque entreprise possède son "profil de partenaires » spécifique. Aussi, avons-nous réalisé une analyse en composantes principales $(\mathrm{ACP})^{9}$ sur l'ensemble des notes et les critères \%crit-ecodef, \%crit-ecoconf et \%crit-ecosens. Cette ACP a été interprétée grâce à la méthode proposée par Lebart, Morineau et Piron (1997) : la première étape consiste à observer les valeurs propres (ou inertie) de chaque axe pour en sélectionner les axes possédant les «pouvoirs explicatifs » les plus élevés. Ainsi,

9. Notre ACP est réalisée sur une matrice de 47 individus et 17 caractéristiques. L'exemple d'application de l'ACP proposé par Lebart porte sur 27 individus et 16 variables (Lebart, Morineau et Piron, 1997). L'utilisation de l'ACP semble donc justifiée dans notre cas. 
nous avons sélectionné, pour interpréter les notes attribuées par les entreprises à leurs partenaires, les trois premiers axes qui ont respectivement des taux d'inertie (pouvant être assimilés à la part d'information portée par l'axe par rapport à l'information totale contenue dans la matrice) de $20 \%, 11 \%$ et $10 \%$. Lebart, Morineau et Piron (1997) conseillent d'interpréter ensuite successivement chaque axe par rapport aux variables ayant les coordonnées les plus proches de 1 sur chaque axe. Le premier plan nous permet d'observer simultanément les deux premiers axes (figure 4).

FiguRE 4

\section{Variables continues actives (niveau de relations avec les partenaires) et illustratives (\% crit-ecodef, \% crit-ecoconf et \% crit-ecosens) dans le premier plan}

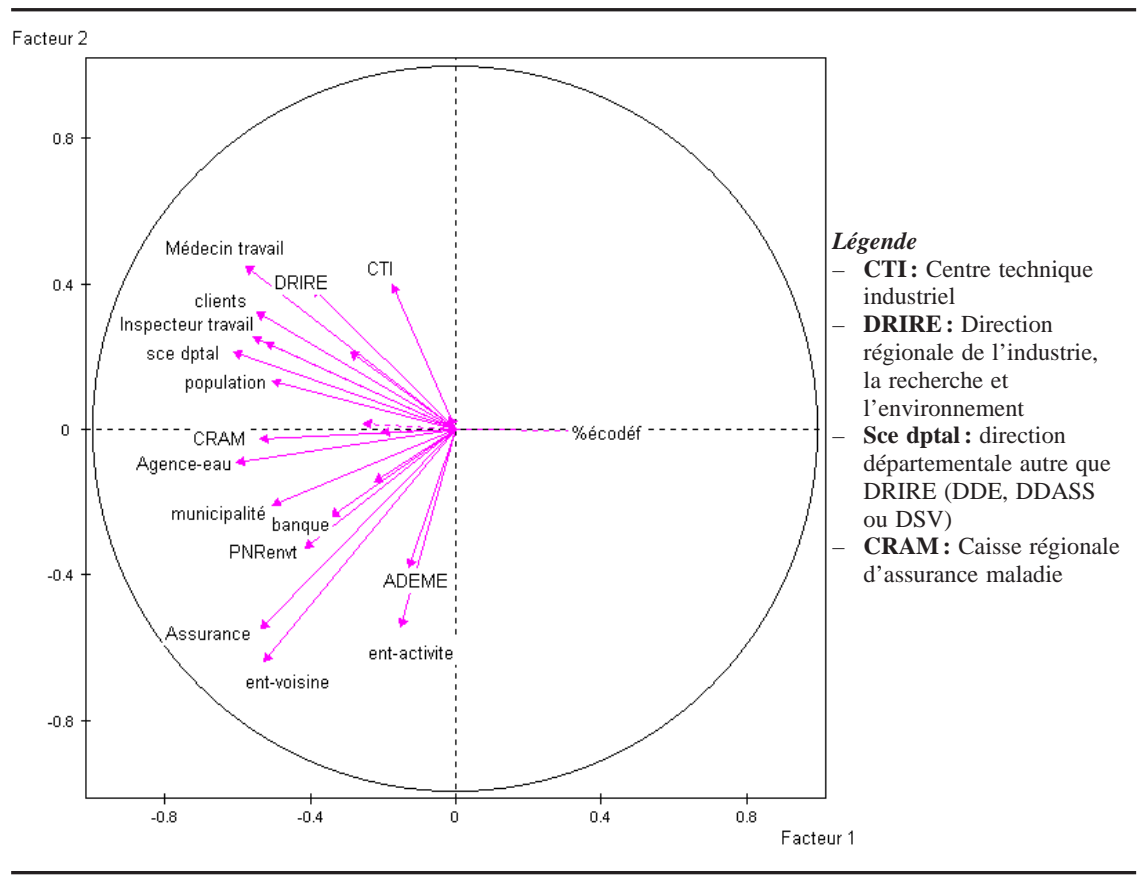

Toutes les notes sont corrélées négativement avec le premier axe (qui représente $21 \%$ de l'information) $)^{10}$. Ce « facteur de taille» (Lebart, Morineau et Piron, 1997) montre que les entreprises qui ont tendance à être ouvertes sur un partenaire

10. Pour plus de clarté, nous n'avons représenté que les notes correspondant à l'environnement, mais le facteur de taille est identique si l'on prend en compte toutes les notes. 
le sont pour l'ensemble de leurs partenaires. La seule variable qui est corrélée positivement avec cet axe est le pourcentage de critères écodéfensifs par rapport au nombre total de critères applicables. Le premier axe oppose donc les entreprises accordant de l'importance à leurs partenaires (situées du côté négatif de l'axe) à celles qui possèdent beaucoup de critères pouvant être qualifiés « d'écodéfensifs » (c'est-à-dire n'intégrant pas la dimension environnementale). Ainsi, plus une entreprise a des relations avec d'autres acteurs, mieux elle respecte l'environnement. Nous avons cherché les caractéristiques de l'entreprise pouvant influencer son positionnement selon cet axe, et donc le niveau d'ouverture de la PME sur ces partenaires. Cependant, parmi les variables prises en compte dans notre questionnaire, aucune modalité de variable nominale ne semble avoir une position significativement différente de l'ensemble de l'échantillon (aucune modalité n'a, sur le premier axe, de valeur test supérieure à 2 , seuil proposé par Lebart, Morineau et Piron, 1997). Ainsi, nous n'avons pas pu mettre en évidence de critère nominal caractéristique de l'ouverture d'une PME/PMI sur ses partenaires.

FIGURE 5

\section{Représentation des variables actives (niveau de relations avec les partenaires) dans le plan constitué par les axes 2 et 3}

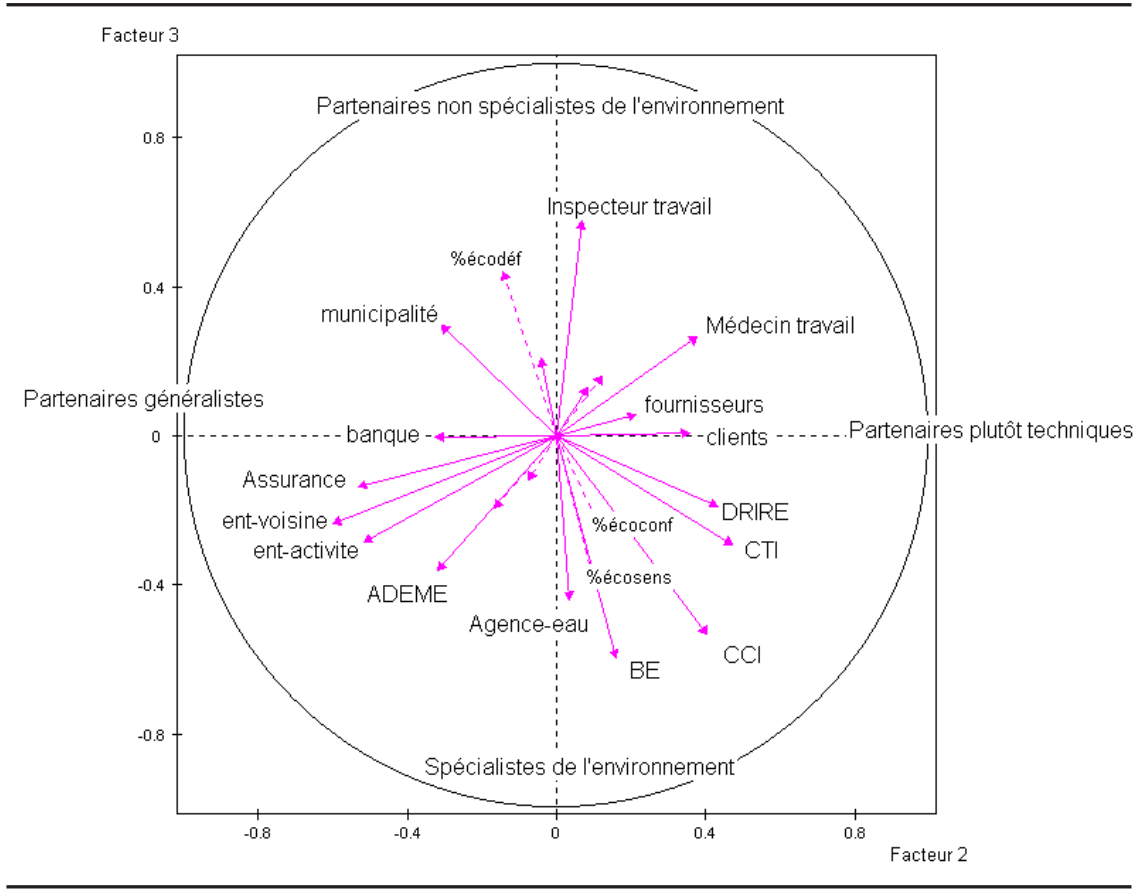

Revue internationale P.M.E., vol. 16, $\mathrm{n}^{\circ}$ 2, 2003 
L'axe 2 oppose, du côté positif, des partenaires avec lesquels l'entreprise a des relations plutôt techniques (centres techniques industriels ou CTI, chambre de commerce et d'industrie ou CCI et DRIRE) à des partenaires « généralistes », situés du côté négatif : assurances, entreprises voisines (ent-voisines) et de même activité (ent-activité). Le pourcentage des critères écosensibles est situé du côté positif de cet axe tandis que celui des critères écodéfensifs est positionné du côté négatif. Cela indique que les entreprises privilégiant les relations avec des partenaires techniques ont tendance à avoir un comportement de type «écosensible » (considérant l'environnement comme un atout) tandis que celles ayant seulement des relations avec des partenaires généralistes ont tendance à être écodéfensives. Si l'on observe les modalités des variables nominales qui ont des valeurs tests supérieures à 2 , il apparaît que, du côté positif de l'axe, se situent plutôt les entreprises interrogées dans la région de $\mathrm{Pau}$, celles qui sont implantées en milieu urbain, et qui possèdent un effectif de 50 à 100 salariés. Du côté négatif de l'axe sont plutôt situées les entreprises rurales, interrogées dans le Pilat, soumises à déclaration selon la législation des installations classées pour l'environnement (ICPE) et en cours de certification qualité. Ainsi, les critères qui influencent le plus le positionnement des entreprises par rapport à cet axe sont le territoire d'implantation, le milieu (urbain ou rural), le statut ICPE et celui relatif à la certification qualité. Cependant, nous n'avons pu établir de lien avec les performances financières de l'entreprise.

Ces constatations, bien que l'on doive tenir compte du biais introduit par l'enquêteur (membre de la CCI dans le cas de Pau, et chercheur de l'École des mines dans le cas du Pilat), font ressortir l'importance du territoire au niveau des relations entretenues par l'entreprise avec ses partenaires. En effet, les entreprises du Pilat sont situées dans un Parc naturel régional, qui s'efforce d'encourager les échanges entre les entreprises et les autres acteurs du territoire (entreprises voisines, municipalité, etc.) tandis qu'à Pau le tissu industriel est centré sur l'aéronautique qui exige une technicité importante.

Le troisième axe oppose des partenaires qui ne sont pas spécialistes de l'environnement mais que les entreprises considèrent comme susceptibles de les aider sur certains thèmes (réglementation générale pour l'inspecteur du travail, émissions atmosphériques diffuses et bruit pour le médecin du travail) à des partenaires spécialistes de l'environnement (CCI, bureau d'études ou BE, agence de l'eau, ADEME). La variable « ratio de critères écodéfensifs » est corrélée positivement (c'est-à-dire du côté des «non-spécialistes » de l'environnement) avec cet axe $(0,44)$ tandis que la variable «ratio de critères écosensibles» est corrélée négativement (-0,37; c'est-à-dire du côté des «spécialistes » de l'environnement). Cela insinue que les entreprises ayant de fortes relations avec des «spécialistes de l'environnement» sont celles qui intègrent le mieux l'environnement. 
Soulignons cependant que les axes 2 et 3 portent chacun la moitié moins d'inertie que l'axe 1 qui est donc le plus représentatif. Les conclusions apportées par ces axes secondaires sont alors statistiquement moins flagrantes que l'interprétation tirée de l'axe 1.

Le lien entre les performances environnementales de l'entreprise et son ouverture sur ses partenaires extérieurs peut s'expliquer par le fait que le contact direct entre parties intéressées et industriels réduit l'asymétrie d'information et, par conséquent, la tentation d'opportunisme. Les entretiens avec les chefs d'entreprise nous ont permis de déceler plusieurs facteurs déterminant l'importance qui sera accordée par l'entreprise à la demande ou au conseil de la partie intéressée :

- l'influence de la partie intéressée sur les performances économiques de l'entreprise ;

- le degré de confiance existant entre la partie intéressée et le chef d'entreprise ;

- la sensibilité personnelle du chef d'entreprise aux problèmes environnementaux;

- la capacité de l'entreprise à améliorer ses performances environnementales : poids des investissements à mettre en œuvre, capacité à introduire des changements organisationnels et techniques, résistance au changement des employés ;

- la perspective des bénéfices économiques pouvant être obtenus à court terme grâce à l'intégration de l'environnement.

\section{Conclusion}

Nous avons montré, grâce à une enquête réalisée auprès de 47 entreprises, que les PME n'ont pas une maîtrise suffisante de l'information environnementale pour avoir le comportement que les instruments traditionnels de régulation des externalités environnementales (approches réglementaire, économique et contractuelle) sont censés les inciter à adopter. Il apparaît que l'accès à l'information est une condition nécessaire, mais non suffisante à l'amélioration de leurs performances environnementales. Notre enquête révèle qu'il n'existe pas de partenaire unanimement privilégié par toutes les PME et qui pourrait être désigné comme le «meilleur canal d'information environnementale». En revanche, il semble que les organisations auxquelles les PME accordent le plus d'importance sont celles avec lesquelles elles sont en contact direct. Par ailleurs, si une entreprise est ouverte sur un de ses partenaires, elle a tendance à l'être sur de nombreux autres acteurs et son niveau de prise en compte de l'environnement sera plus élevé que celui des

Revue internationale P.M.E., vol. 16, nº 2, 2003 
entreprises n'accordant pas d'importance aux organisations extérieures. Nous avons également mis en évidence le fait que le territoire est l'un des principaux critères qui déterminent le type de partenaires qui sera privilégié par l'entreprise.

Ainsi, pour inciter les PME / PMI à améliorer leurs performances environnementales, il semble pertinent de promouvoir les relations entre entreprises et ses parties intéressées. Les acteurs locaux pourraient animer des programmes de sensibilisation à l'environnement visant à stimuler ces contacts directs et impliquer les partenaires les plus influents sur les entreprises. En outre, les institutions nationales pourraient mettre en place, parallèlement aux politiques environnementales réglementaires et économiques traditionnelles, une «politique informationnelle» visant à stimuler la régulation par les réseaux afin de promouvoir les échanges portant sur les questions environnementales entre PME et acteurs locaux publics et privés. Collectivités locales, organisations consulaires, associations d'industriels, centres techniques, partenaires économiques traditionnels, etc., peuvent agir comme « relais d'information environnementale » au sein de partenariats et dans le cadre de programmes collectifs. L'outil de diagnostic que nous proposons peut être utilisé pour établir les besoins des entreprises et leurs « profils de partenaires » afin de déterminer les thèmes à aborder par ces programmes ainsi que les organismes-relais les mieux adaptés au territoire concerné.

\section{Bibliographie}

AGGERI, F. (1999), «Environmental policies and innovation: a knowledge-based perspective on cooperative approaches », Research Policy, vol. 28, n 7, p. 699-717.

BÖRKEY, P. et M. GLACHANT (1999), Les accords volontaires négociés : une analyse de leur efficacité, Étude réalisée pour le ministère de l'Aménagement du territoire et de l'Environnement et l'ADEME.

BRODHAG, C. (1994), « Pour concilier l'entreprise et l'environnement, il faut une approche systémique », Annales des mines, p. 41-44.

Butel-Belini, B. (1997), L'intégration de la donnée écologique dans la gestion de l'entreprise: une analyse contingente au niveau des sites de production, Thèse pour le doctorat en sciences de gestion, Université de Lille I.

COMMISSION ÉCONOMIQUE DES NATIONS UNIES POUR L'EUROPE - CENUE (1998), Convention sur l'accès à l'information, la participation du public au processus décisionnel et l'accès à la justice en matière d'environnement. ECE/CEP/43, disponible sur Internet : URL : <http://www.unece.org/env/europe/ppconven.htm>.

DE BAKER, P. (1999), L'impact économique et l'efficacité environnementale de la certification ISO 14001/EMAS des entreprises industrielles. Étude financée par le service économie 1'ADEME disponible sur Internet : 〈http://www.ademe.fr/>.

FAUCHEUX, S. et J.F. NOËL (1995), Économie des ressources naturelles et de l'environnement, Paris, Armand Collin.

Revue internationale P.M.E., vol. 16, $n^{\circ}$ 2, 2003

(C) 2003 - Presses de l'Université du Québec 
GODARD, O. (1993), « Stratégies industrielles et conventions d'environnement : de l'univers stabilisé aux univers controversés », INSEE - Méthodes Environnement et économie, $\mathrm{n}^{\text {os }} 39-40$, p. $145-174$.

GONDRAN, N. (2001), Système de diffusion d'information pour encourager les PME/ $P M I$ à améliorer leurs performances environnementales, Thèse de doctorat en sciences et techniques du déchet, ENS des mines de Saint-Étienne, novembre, disponible sur Internet: 〈http://www.agora21.org/entreprise/these2.html>, p. 165.

Gondran, N. et C. Brodhag (2000), «Modèle de prise de décision et d'utilisation de l'information environnementale pour l'intégration de l'environnement par la PME/ PMI », Colloque international francophone sur la PME, Lille: <http://www. airepme.univ-metz.fr/>.

Gondran, N., P. Formisyn, I. Ensuque, X. Boespflug et C. Brodhag (2000), «Proposition d'une méthode de pré-diagnostic environnemental basée sur l'accès à l'information: Application à des entreprises tunisiennes », Déchets - Sciences et Techniques, $\mathrm{n}^{\circ} 18$, p. 39-45.

Hillary, R. (éd. 2000), Small and Medium-sized Enterprises and the Environment, Sheffield, Greenleaf Publishing.

ISO (1996), Systèmes de management environnemental - Spécifications et lignes directrices pour son utilisation, norme européenne - norme française NF EN ISO 14001. AFNOR, p. 2.

JULIEN, P.-A. (dir.) (1997), Les PME: bilan et perspectives, GREPME (Groupe de recherche en économie et gestion des PME), Paris, Economica.

Koenig, G. (1993), Les théories de la firme, Paris, Economica, p. 65.

Lebart, L., A. Morineau et M. PIRON (1997), Statistique exploratoire multidimensionnelle, Paris, Dunod, p. 57.

PERSONNE, M. (1998), Contribution à la méthodologie d'intégration de l'environnement dans les PME-PMI: Évaluation des performances environnementales, Thèse de doctorat en sciences et techniques du déchet, École nationale supérieure des mines, disponible sur Internet : 〈http://www.agora21.org/entreprise/index1.html>.

QUIVY, R. et L. VAN CAMPENHOUDT (1995), Manuel de recherche en sciences sociales, Paris, Dunod, p. 192.

REYNAUD, E. (1997), Les déterminants du comportement de protection de l'environnement des entreprises, Thèse pour le doctorat en sciences de gestion, Université de droit et des sciences d'Aix-Marseille III, p. 136.

SimON, H. (1955), «A behavioral model of rational choice», Quarterly Journal of Economics, vol. 69, p. 99-118.

Revue internationale P.M.E., vol. 16, $\mathrm{n}^{\circ}$ 2, 2003

(C) 2003 - Presses de l'Université du Québec 